\title{
Osteopathy and Emergency: A Model of Osteopathic Treatment Aimed at Managing the Post-Traumatic Stress-Brief and Useful Guide-Part 2
}

\author{
Luca Collebrusco1,2, Simone Fabri², Antonio Furfaro' ${ }^{2}$, Ilaria Tanini' ${ }^{2}$, Rita Lombardini', \\ Andrea Rizza², Paolo Zavarella² \\ ${ }^{1}$ Rehabilitation Unit, National Health Service of Umbria, Perugia, Italy \\ ${ }^{2}$ Research Department CROMON, EDUCAM School, Rome, Italy \\ ${ }^{3}$ Department of Medicine, University of Perugia, Perugia, Italy \\ Email: luca.collebrusco@unipg.it
}

How to cite this paper: Collebrusco, L., Fabri, S., Furfaro, A., Tanini, I., Lombardini, R., Rizza, A. and Zavarella, P. (2018) Osteopathy and Emergency: A Model of Osteopathic Treatment Aimed at Managing the Post-Traumatic Stress-Brief and Useful Guide-Part 2. Health, 10, 1597-1608. https://doi.org/10.4236/health.2018.1011120

Received: October 19, 2018

Accepted: November 24, 2018

Published: November 27, 2018

Copyright $\odot 2018$ by authors and Scientific Research Publishing Inc. This work is licensed under the Creative Commons Attribution International License (CC BY 4.0).

http://creativecommons.org/licenses/by/4.0/

\begin{abstract}
This work aims to describe a well standardized therapeutic path in reference to the article "Osteopathy and Emergency: A Model of Osteopathic Treatment Aimed at Managing the Post-Traumatic Stress-Part 1 in the form of a practical guide for manual therapist, Open Journal of Therapy and Rehabilitation, Vol. 10, No. 7, July 2018, https://doi.org/10.4236/health.2018.107074". The osteopathic manipulative treatment (OMT) approach we propose can be complementary to the psychological therapy in use today, and consists of ten standardized techniques directed to the anatomical structures more involved in the stress answer. This protocol was designed to improve and sustain the management of subjects exposed to extraordinary exogenous stress or post-traumatic stress disorder patients (PTSD).
\end{abstract}

\section{Keywords}

Post-Traumatic Stress Disorder, Osteopathic Manipulative Treatment, Visceral Osteopathic, Osteopathy Cranial Field, Myofascial and Musculoskeletal Techniques

\section{Introduction}

The Fascia is defined by Still as "the place to look for the cause of disease and the place to consult and begin the action of remedies in all diseases" [1]. The osteopathic manipulative treatment (OMT) consists of a range of direct, indirect, 
combined, fluid, and reflex-based manual techniques (Table 1) [2] that are applied to joints, muscles and especially to fascia, a specialized connective tissue that provides body tensegrity.

It is ubiquitously in the human body, serving mechanical, metabolic and immunological functions. Due to the wide scope of application, the osteopath selects a specific approach model based on the patient's needs. Once identified the nature of somatic dysfunction (SD), specific techniques of the OMT can be applied. In our case, the loss of the general equilibrium of the verticality causes an increase of the allostatic load addressed to musculoscheletal system. We know that the General Osteopathic Treatment (GOT) is well received in patient, and the degree of acceptance of the body has been increased according to the best competences of osteopathy [3]. Furthermore this document proposes an osteopathic treatment plan designed to reduce physical symptoms associated with post-traumatic stress disorder (PTSD) and cooperate in managing PTSD in tiling to today's best standard in psychological support methods [4] [5]. All the selected techniques will be classified according to the type of manipulative procedure as described in literature [6] [7] [8] and will be explained in detail.

\section{OMT for PTSD}

Each treatment lasted 45 minutes. At the beginning of each treatment, the operator evaluates the patient through a maximalist (GOT) and minimalist (SD) osteopathic model. The treatment maneuvers with their procedural description are shown below.

\subsection{Osteopathy in the Cranial Field (CR): Frontal Lift}

Objective: Rebalancing the dysfunctions of the frontal bones with an action at a sutural level (fronto-parietal, front nasal suture) and relaxation of the duramater.

Table 1. Overview of OMT Protocol for PTSD.

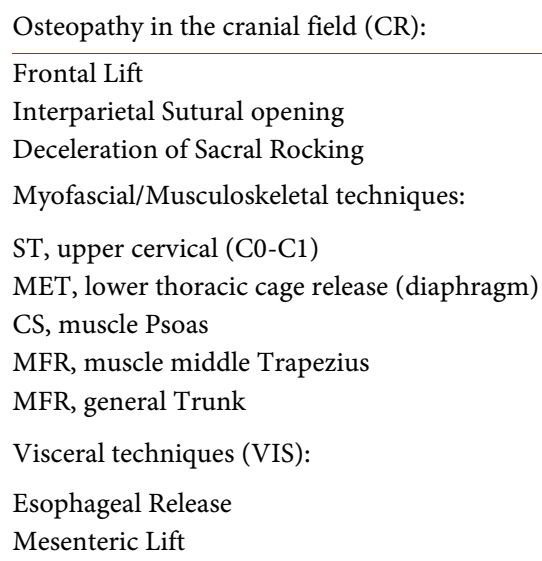

Abbreviation: OMT, Osteopathic Manipulation Treatment. PTSD, post-traumatic stress disorder. CR, osteopathy in the cranial field. ST, soft tissue treatment. MET, muscle energy treatment. CS, counterstrain treatment. MFR: myofascial release technique. VIS, visceralmanipulative treatment. 
Maneuver procedure: Patient supine, the therapist is sitting with both forearms resting on the bed, acting as a fulcrum. Place both hypotenar eminences on the orbital pillars of the frontal bones and the tenar ones anteriorly and laterally to the coronal suture, intertwining the fingers over the metopic suture. The tenar and hypotenar eminences of the operator exert a gentle compression in the medial direction to disengage the front-parietal sutures, and then induce an internal rotation of the frontal bones while at the same time exert anterior drive. This position is maintained until the operator perceives an external rotation of the frontal bones (feeling an expansion under the hypotenar eminences). The technique is concluded by accompanying the frontal in a neutral position and re-evaluating the parameters of the primary respiratory mechanism (Figure 1), [9] [10] [11].

\subsection{CR: Interparietal Sutural Opening}

Objective: To restore the motion of the sagittal suture, increasing the drainage of the upper sagittal sinus and re-launching the fluctuation of the cerebrospinal fluidtrough the stimulus on the granulation of Pacchioni.

Maneuver procedure: Patient supine, the therapist is sitting with both forearms resting on the bed, acting as a fulcrum. Cross the thumbs on the sagittal suture of the patient, anteriorly and superiorly to Lambda. The rest of the operator's fingers rest on the lateral surfaces of the patient's parietal bones. Using the thumbs, the operator induces an external rotation of the parietal bones, following the entire course of the sagittal suture, in order to decompress it. The technique ends when the operator feels a sensation of softening, heating or an increase in fluid movement. The technique ends by re-evaluating the parameters of the primary respiratory mechanism (Figure 2), [9] [12].

\subsection{Soft Tissue (ST): Upper Cervical C0-C1}

Objective: To reduce under-occipital myofascial tensions and to obtain a neurovegetative inhibition.

Maneuver procedure: Patient supine, the osteopath positions the fingertips in the under-occipital region of the patient with the palms facing upwards, making contact with the upper portion of the trapezius and the underlying musculature. The osteopath slowly and gently applies a tissue tension upwards for a few seconds and then releases after about a minute in order to obtain an inhibitory effect at a neurovegetative level. This pressure can be reapplied and released slowly and rhythmically until the operator senses a release in the tissue's tension (Figure 3), [13] [14].

\subsection{Visceral Manipulative Treatment (VIS): Esophageal Release}

Objective: To decrease the tensions along the course of the esophagus by modulating the vagal stimulation.

Execution of the Maneuver: Patient on the back, the operator standing at the 
side of the patient at the level of the thorax. With the cephalic hand, the operator takes support with the thumb grip on the tracheal cartilage to take contact with the esophagus. The operator asks the patient for a swallowing to verify the correct localization. The osteopath takes support with the caudal hand at about 2 - 3 $\mathrm{cm}$ below and left from the xiphoid process on the patient's left hemithorax. The operator exercises a light cephalic traction with the cranial hand and a caudal traction with the other, in a slightly diagonal left direction. Perform the maneuver according to the diaphragmatic breathe, increasing traction in the inspiratory phase and keeping it in the expiratory phase, for $3-4$ respiratory cycles and then gently release the grip (Figure 4), [14] [15] [16].

\subsection{Muscle Energy Treatment (MET): Lower Thoracic Cage Release (Diaphragm)}

Objective: Release the tensions of diaphragmatic musculature to re-launch the respiratory function, restore correct intercavitary pressure and increase venous and lymphatic return.

Maneuver procedure: Patient supine, the operator is positioned right lower to the inferior rib profile with the thumbs placed in cephalic direction, listening to the fascial movement. Once the direction of greater rotational limitation has been established, it performs the same evaluation on the latent right and left flexion. Once the two restriction positions have been established, stack the parameters, keeping them still for 3 - 4 patience's breaths until a release is obtained (Figure 5), [17] [18] [19].

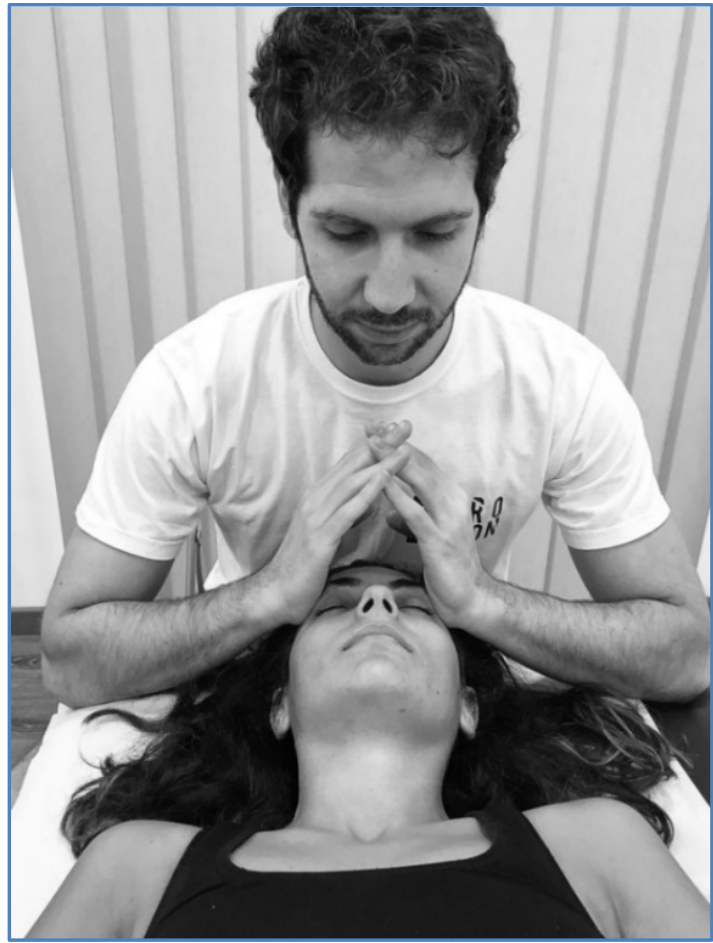

Figure 1. Osteopathy in the cranial field (CR): Frontal Lift. 


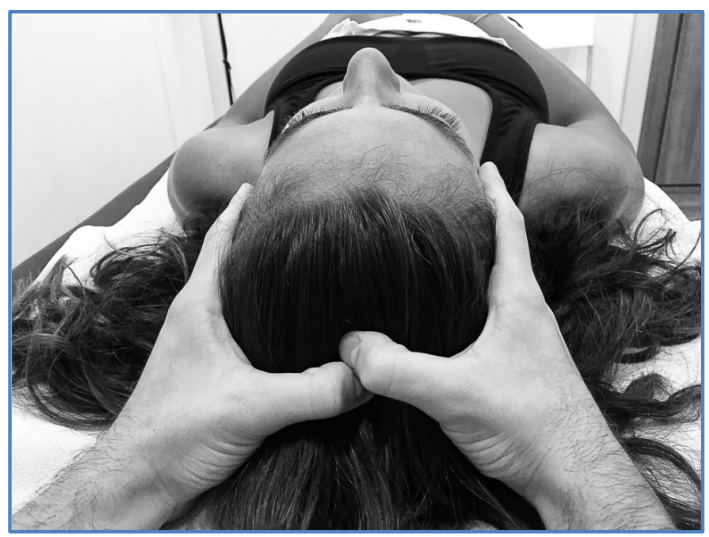

Figure 2. Osteopathy in the cranial field (CR): Interparietal Sutural Opening.

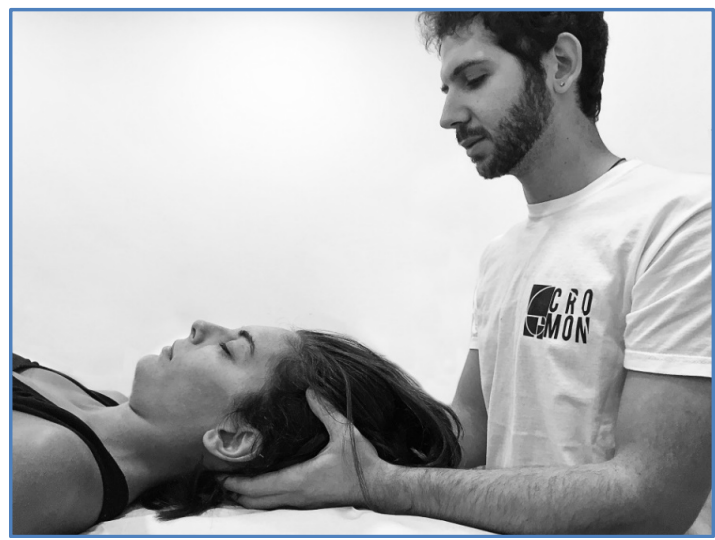

Figure 3. Soft Tissue (ST): Upper cervical C0-C1.

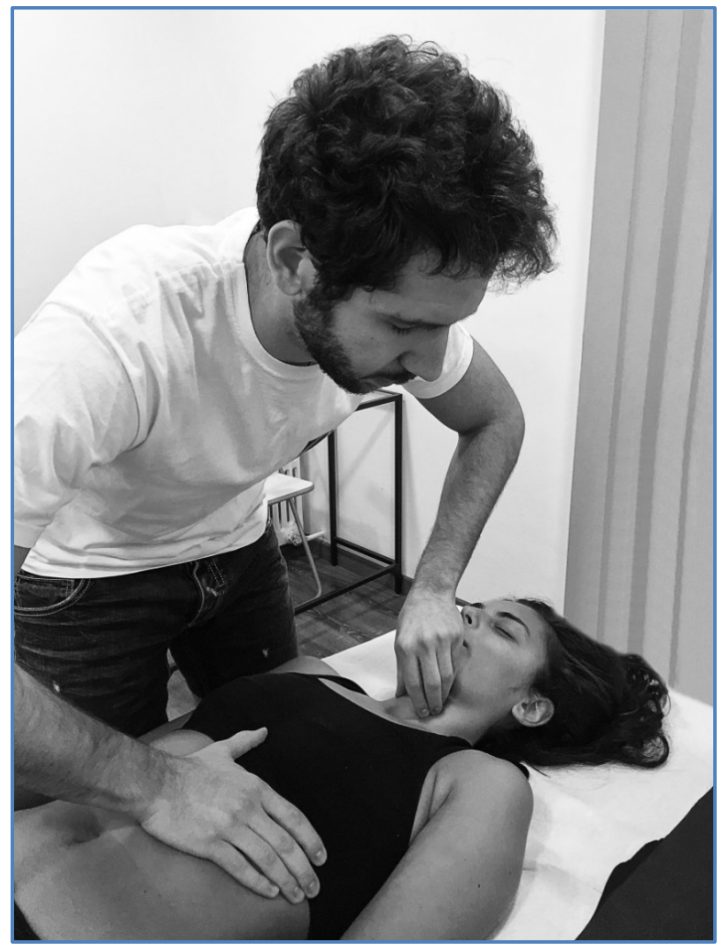

Figure 4. Visceral manipulative treatment (VIS): Esophageal release. 


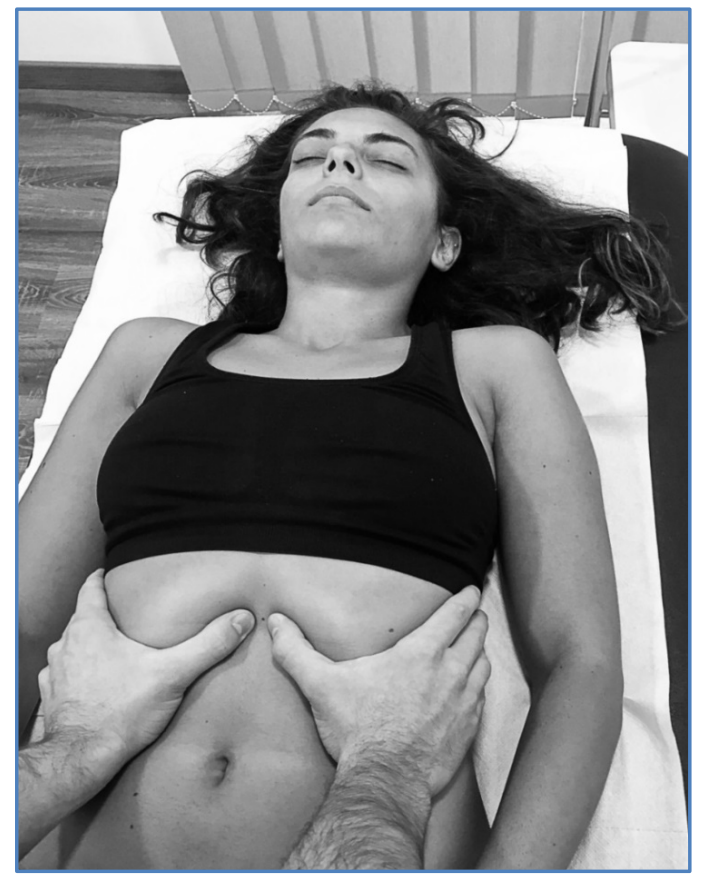

Figure 5. Muscle Energy Treatment (MET): Lower Thoracic Cage Release (Diaphragm).

\subsection{Counterstrain Treatment (CS): Muscle Psoas}

Objective: To reduce pelvic pain through the lombar plexus by relasing the ileo-psoas.

Maneuver procedure: Patient on the back, the operator standing on the side of the psoas to be treated, the external hand contact the ileo-psoas muscle, taking two inches medially and slightly below the anterior superior iliac spine and finding the tender point in depth. Once the painful area is found, it flexes the hip 90 degrees with the inner hand. It modulates the pressure of the tender area with an intensity of 10/10 with the pressure carried out until it reaches a decrease in pain intensity of about $2 / 10$. Holds the position for 90 seconds and then places the leg passively and slowly into neutral position, repeat the maneuver twice. Re-evaluate the tender point to check the decrease in pain (Figure 6), [14] [17] [20] [21].

\subsection{VIS: Mesenteric Lift}

Objective: To reduce the congestion of the abdominal region, facilitating lymphatic and venous drainage, to reduce the tension of the lumbar spine and relative plexus.

Maneuver procedure: Patient in lateral decubitus, the operator positions the fingers of both hands transversally in projection the mesentery, in the middle of the line between the navel and the ASIS. Apply a slight push towards the pubic symphysisto the visceral package, and, in accordance with the breath, listen to the tissue response until is possible to feel its return. Repeat the maneuver 2 - 3 times, in order to reduce tissue's tension (Figure 7), [17] [22] [23]. 


\subsection{Myofascial Release Technique (MFR): Muscle Middle Trapezius}

Objective: Reduce the thoracic myofascial restrictions to relieve the pain in the scapular girdle.

Maneuver procedure: Prone patient, therapist standing at the head of the patient, arms crossed with the fingers of the hand facing outwards and with the two tenar and hypotenar eminences of each hand at the level of the medial side of the scapula. Through a slight push of the pectorals along the direction of the fingers of the two hands in support, listening to the diaphragmatic breathing of the patient, in the expiratory phase makes a slight push and in the inspiratory phase keeps the thrust given for 3 - 4 respiratory times and then release the position (Figure 8), [21].

\subsection{MFR: General Trunk}

Objective: To decrease the fascial restrictions along the axis of the spine.

Maneuver procedure: patient prone, the operator standing on the right side of the patient, contacts the area under the occipital bone with the caudal hand, with the cranial hand he takes support at the level of the sacral bases with the fingers in the direction of the coccyx. Performer the tecnique while listening to the diaphragmatic breathing, during the expiratory phase give a slight push with the pectoral muscles towards the floor and with a cranial hand give a downward thrust, at the same time with the caudal hand give a push upwards. Keep the thrust for 3 - 4 respiratory times and then release the position (Figure 9), [16] [21].

\subsection{CR: Deceleration of Sacral Rocking}

Objective: To stimulate parasympathetic activity.

Maneuver procedure: Prone patient, osteopath on the side of the patient. Place both hands on top of the sacrum. Ask the patient for a slow and deep breathing. During inspiration, push the sacral apices anteriorly, during expiration encourage the sacral flexion by pushing the sacral base anteriorly. Repeat the maneuverfor 3 - 4 respiratorycycles. (Figure 10), [17] [21].

The administered protocol aims to improve and support the management of subjects exposed to extraordinary exogenous stresses, such as the earthquake occurred to the population we had the chance to treat. The totality of the sample examined presented a series of common symptoms such as: recurring headaches, cervical pain, joint pain in the scapular girdle, low back pain, generalized muscle tension, gastric reflux, irritable bowel syndrome and constipation. The presented vegetative symptomatology has oriented us towards an osteopathic model which proposes to integrate techniques coming from different osteopathic backgrounds in order to respond in a soft way to this clinical picture. The integration between the minimalist (SD) and maximalist (GOT) approach [22] allowed us to combine the five osteopathic models [5] through an indirect approach modality typical of myofascial treatment. 


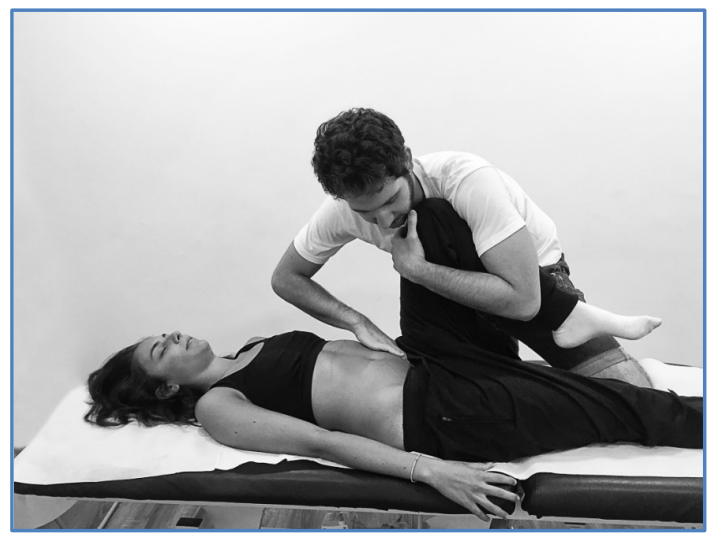

Figure 6. Counterstrain Treatment (CS): Muscle Psoas.

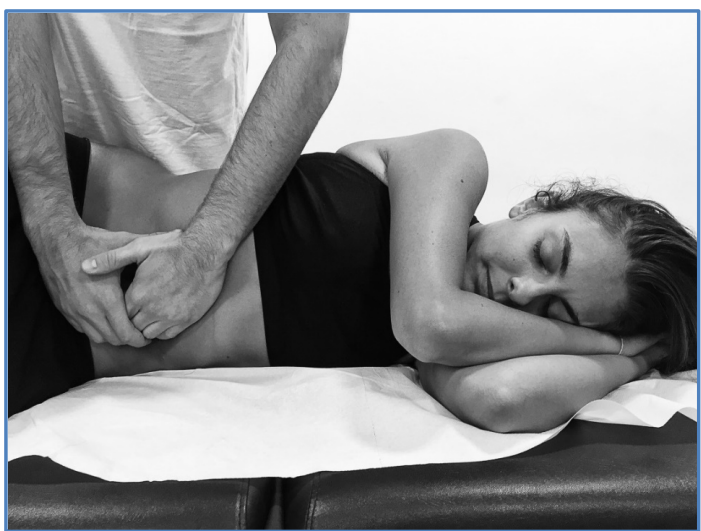

Figure 7. Visceral Manipulative Treatment(VIS): Mesenteric Lift.

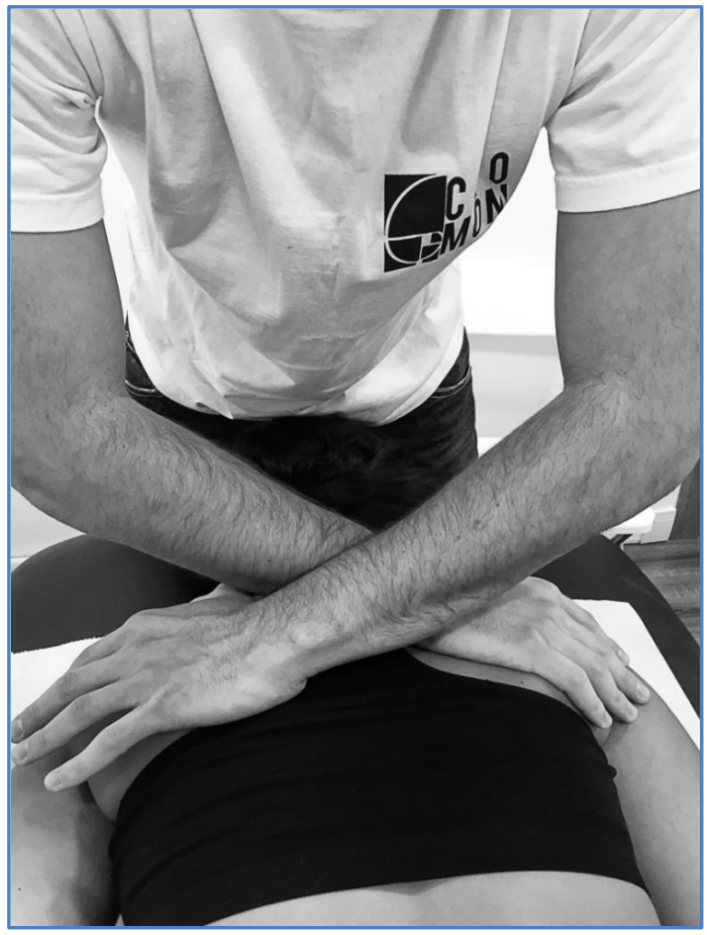

Figure 8. Myofascial Release Technique (MFR): Muscle Middle Trapezius. 


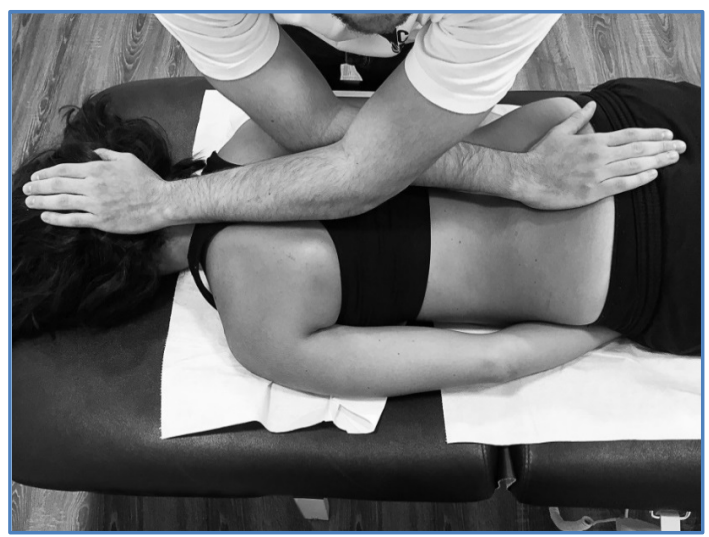

Figure 9. Myofascial Release Technique (MFR): General Trunk.

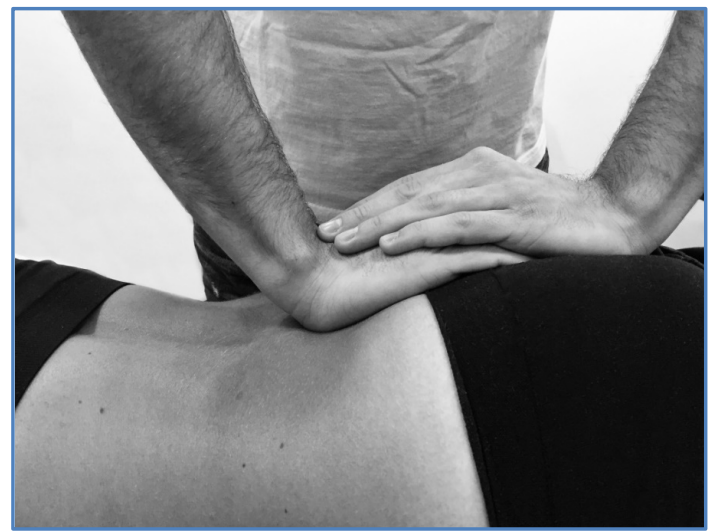

Figure 10. Osteopathy in the cranial field (CR): Deceleration of Sacral Rocking.

\section{Discussion}

Since ostheopatic philosophy and practice is based on the body's innate ability to self-regulate, we believe that SD can alter muscle tone, generate a contracture and modify the elasticity/compliance of soft tissues and interfere with physiological and mental processes leading to emotional changes [23].

From the analysis of the literature is evident how the OMT can play a decisive role in helping the patients in case of extraordinary stress, potentially able to determine a symptomatic picture as the one of PTSD. As widely described by many qualified authors, osteopathic manipulation, stimulating parasympathetic activity [24] can also influence the neuroendocrine system [25], and give a very important help in this field of application by the rebalancing the Hypothalamic Pituitary Adrenal axis (HPA), with consequent outcome of anti-inflammatory and anti-pain reaction in the tissues. We believe that this approach can fasten the remodulation of the central hyper-reactivity related to a dysfunctional response of the system, in its physical, mental and emotional components. The OMT, aims to prevent the negative responses of the patient's body in order to obtain a health-friendly environment as perfectly as possible [26]. OMT in collaboration with other medical figures, can play a relevant role in the management of strong stress conditions and in the prevention of PTSD in patients involved in traumas 
resulting from environmental disasters or determined by man, as we experience in Amatrice, Umbria (Italy), $24 \mathrm{~h}$ August 2016 where this approach has been tested in a pilot study. The data we collected are still in progress of evaluation. We hope that, even if the sample examined is limited, our encouraging results could be a first step towards a complete OMT protocol able to improve and support the management of subjects exposed to PTSD, since this approach made patients, already from the first session, to report us a feeling of general well-being and relaxation of muscular tension.

\section{Conclusions}

When the body system has to face an extraordinary stress, the allostatic load increases and the potential for self-regulation is affected: for this reason, an approach like the osteopathic one, which is not invasive and directed to the stimulation of those natural innate forces in the body, can be considered useful in the approach to disorders such as post-traumatic stress syndrome.

Osteopathy, with its gentle approach, can modulate almost every aspect related to the human body and each system that regulates it on a musculoskeletal, neurological, visceral and emotional level, in order to release, can alter allostatic overload. This short guide could represent a promising start in the research of a complementary strategy to help patients with PTSD. It is suggested to combine our manual treatment protocol with conventional methods already in use in emergency situations to relieve the shock to which the people were exposing [22].

\section{Acknowledgements}

We would like to thank EDUCAM School to make the research project possible, Dr. Oriana D'Emidio, coordinator of the Amatrice health care assistance unit, the diocesan CARITAS for the project location, the mayor of Amatrice, all the citizens and the Italian armed forces for support in the emergency period.

\section{Conflicts of Interest}

The authors declare no conflicts of interest regarding the publication of this paper.

\section{References}

[1] Still, A.T. (1892) The Philosophy and Mechanical Principles of Osteopathy. Hudson-Kimberly Publication Co., Kansas City, Missouri, United States (US).

[2] Collebrusco, L., Lombardini, R. and Censi, G. (2017) Regional Interdependence: A Practical Guidance in Rehabilitation Clinic-Part. 2. Open Journal of Therapy and Rehabilitation, 5, 11-28. https://doi.org/10.4236/ojtr.2017.51002

[3] Lombardini, R. and Collebrusco, L. (2016) Osteopathy and Nutrition for Managing the Irritable Bowel Syndrome-Brief and Useful Guide. 1st Edition, Universitas Studiorum S.r.l., Mantova, Italy.

[4] Lunghi, C., Baroni, F. and Alò, M. (2017) Il ragionamento clinico osteopatico. Edizioni Edra S.p.A., Italy.

[5] Tozzi, P., Lunghi, C. and Fusco, G. (2005) I cinque modelli osteopatici. Razionale, 
applicazione, integrazione. Dalla tradizione all'innovazione per un'osteopatia incentrata sulla persona. Ed. Edra S.p.A., Italy.

[6] Greenman, P.E. (1996) Principles of Manual Medicine. Williams \& Wilkins, Baltimore, Michigan, United States (US).

[7] Robert Boyles, P.T., Timothy, W., Flynn, P.T., Julie, M., Whitman, P.T., Robert, S., Wainner, P.T., Paul, E. and Mintken, P.T. (2012) Spinal \& Extremity Manipulation. The Basic Skill Set. 2nd Edition, EIM (Evidence in Motion), Louisville, Kentucky, United States (US).

[8] Glossary of Osteopathic Terminology, Prepared by the Educational Council on Osteopathic Principles (ECOP) of the American Association of Colleges of Osteopathic Medicine (AACOM), Revised November, 2011.

[9] Ward, R. (2003) Foundations for Osteopathic Medicine. 2nd Edition, Lippincott Williams \& Wilkins, Philadelphia.

[10] Arbuckle, E. (1997) The Selected Writings of Beryl. National Osteopathic Institute and Cerebral Palsy Foundation.

[11] Weaver, C. (1936) The Cranial Vertebrae. The Journal of the American Osteopathic Association, 35, 328-336.

[12] Magoun, H. (1976) Osteopathy in the Cranial Field. 3rd Edition, Northwest Printing, Boise.

[13] Di Giovanna, E. and Schiowitz, S. (2005) An Osteopathic Approach to Diagnosis and Treatment. Lippincott Williams \& Wilkins, Philadelphia.

[14] Patriquin, D.A. and Ward, R.C. (2003) Foundation for Osteopathic Medicine. 2nd Edition, Williams \& Wilkins Philadelphia, Limpiccot, 1051-1055.

[15] Hebgen, E. (2011) Visceral Manipulation in Osteopathy. Edition Thieme Medical. https://doi.org/10.1055/b-002-79387

[16] Collebrusco, L., Lombardini, R. and Censi, G. (2017) An Alternative Approach to the Gastroesophageal Reflux Disease: Manual Techniques and Nutrition. Open Journal of Therapy and Rehabilitation, 5, 98-106. https://doi.org/10.4236/ojtr.2017.53009

[17] Degenhardt, B.F. and Kuchera, M.L. (1996) Update on Osteopathic Medical Concepts and the Lymphatic System. Journal American Osteopathic Association, 96, 97-100. https://doi.org/10.7556/jaoa.1996.96.2.97

[18] Chaitow, L., Bradley, D. and Gilbert, C. (2002) Multidisciplinary Approaches to Breathing Pattern Disorders. Churchill Livingstone, London, 86, 51.

[19] Collebrusco, L. (2014) Positional Release Techniques as a Diagnostic-Therapeutic Approach in Physiotherapy. Open Journal of Therapy and Rehabilitation, 2, 19-20. https://doi.org/10.4236/ojtr.2014.21004

[20] Nicholas, A.S. and Nicholas, E.A. (2008) Atlas of Osteopathic Techniques. Lippincott Williams \& Wilkins, Philadelphia.

[21] Steel, K.M., et al. (2006) A Pocket Manual of OMT. Osteopathic Manipulative Treatment for Physicians, Lippincott Williams and Wilkins, Philadelphia.

[22] Collebrusco, L., Fabri, S., Furfaro, A., Tanini, I., Lombardini, R., Rizza, A. and Zavarella, P. (2018) Osteopathy and Emergency: A Model of Osteopathic Treatment Aimed at Managing the Post-Traumatic Stress-Part 1. Health, 10, 998-1005.

[23] Collebrusco, L. and Lombardini, R. (2013) Osteopathic Manipulative Treatment and Nutrition: An Alternative Approach to the Irritable Bowel Syndrome. Health, 5, 87-93. https://doi.org/10.4236/health.2013.56A2013 
[24] McGlone, F., Cerritelli, F., Walker, S. and Esteves, J. (2017) The Role of Gentletouch in Perinatal Osteopathic Manual Therapy. Neuroscience \& Biobehavioral Reviews, 72, 1-9. https://doi.org/10.1016/j.neubiorev.2016.11.009

[25] Bottaccioli, F. (2008) Il sistema immunitario: La bilancia della vita. Come è fatto e come funziona in salute e in malattia. EdizioneTecniche Nuove Spa, Milano.

[26] Collebrusco, L. and Lombardini, R. (2014) What about OMT and Nutrition for Managing the Irritable Bowel Syndrome? An Overview and Treatment Plan. EXplore, 10, 309-318. https://doi.org/10.1016/j.explore.2014.06.005

\section{Abbreviation}

CR: Osteopathy in the cranial field

CS: Counterstrain Treatment

GOT: General Osteopathic Treatment

HPA: Hypothalamic Pituitary Adrenal axis

MET: Muscle Energy Treatment

MFR: Myofascial Release Technique

OMT: Osteopathic Manipulative Treatment

PTDS: Post Traumatic Stress Disorder

SD: Somatic Dysfunction

ST: Soft Tissue Treatment

VIS: Visceral Manipulative Treatment 